\title{
MinI-REVIEW
}

\section{Apoptotic regulation and tRNA}

\author{
Yide $\mathrm{Mei}^{1}$, Aaron Stonestrom ${ }^{1}$, Ya-Ming Hou ${ }^{2}$, Xiaolu Yang ${ }^{1 凶}$ \\ ${ }^{1}$ Department of Cancer Biology and Abramson Family Cancer Research Institute, University of Pennsylvania School of \\ Medicine, Philadelphia, PA 19104, USA \\ 2 Department of Biochemistry and Molecular Biology, Thomas Jefferson University, Philadelphia, PA 19107, USA \\ $\triangle$ Correspondence: xyang@mail.med.upenn.edu \\ Received August 19, 2010 Accepted August 26, 2010
}

\begin{abstract}
Apoptotic regulation is critical to organismal homeostasis and protection against many human disease processes such as cancer. Significant research efforts over the past several decades have illuminated many signaling molecules and effecter proteins responsible for this form of programmed cell death. Recent evidence suggests that transfer RNA (tRNA) regulates apoptotic sensitivity at the level of cytochrome $c$-mediated apoptosome formation. This finding unexpectedly places tRNA at the nexus of cellular biosynthesis and survival. Here we review the current understanding of both the apoptotic machinery and tRNA biology. We describe the evidence linking tRNA and cytochrome $c$ in depth, and speculate on the implications of this link in cell biology.
\end{abstract}

KEYWORDS apoptotic regulation, tRNA, cytochrome $c$

\section{CYTOCHROME C AND THE INTRINSIC APOPTOTIC PATHWAY}

Apoptosis is the major process through which unnecessary, damaged, and harmful cells are eliminated. Multi-cellular development, homeostasis, immunity and protection against cancer all require intricate regulation of programmed cell death (Thompson, 1995; Vaux and Korsmeyer, 1999). Two major apoptotic routes exist in mammalian cells: the extrinsic and intrinsic pathways. The final common end point of both pathways is activation of the proteolytic caspase (cysteinedependent aspartate specific protease) cascade. This causes cleavage of numerous cellular targets resulting in cellular shrinkage, fragmentation, membrane blebbing and nuclear condensation and termination of cell life (Chang and Yang, 2000; Li and Yuan, 2008). The extrinsic pathway is activated by engaging death receptors such as Fas and TRAIL receptors with their cognate ligands (Ashkenazi and Dixit, 1998). This leads to the formation of the membrane-bound death inducing signaling complex (DISC), which recruits the initiator procaspase- 8 by the adaptor protein FADD. In the DISC, procaspse-8 is activated by oligomerization (Yang et al., 1998; Chang et al., 2003). The intrinsic pathway responds to "intracellular" cues such as DNA damage, oncogene activation, nutrient deprivation, and lineage information. Intrinsic apoptotic signals converge to cause mitochondrial outer membrane permeabilization (MOMP). This leads to the release of cytochrome $c$, an essential electron transfer chain component, from mitochondria to cytosol (Wang, 2001). The discovery of the role of cytochrome $c$ in apoptosis (Liu et al., 1996) came as a shock to a field that regarded apoptosis as being carried out by deadly signaling molecules. It casts light on an ingenious design during evolution that has endowed a potent destructive power to a molecule so essential for life, and gives a striking physical form for the philosophic phrase that life and death are inseparable.

In the cytosol, cytochrome $c$ is a ligand for Apaf-1 (the apoptotic protease activating factor-1) (Zou et al., 1997). Apaf-1 binds to dATP or ATP in an inactive state, and cytochrome $c$ binding stimulates the intrinsic (d)ATPase activity of Apaf-1 leading to the hydrolysis of (d)ATP to (d)ADP. Subsequently, (d)ADP is exchanged with a free (d)ATP molecule in the cytosol (Kim et al., 2005). Then, with the assistance of at least three proteins: HSP70, cellular apoptosis susceptibility protein (CAS), and putative HLA-DRassociated protein 1 (PHAP1), Apaf-1 assemblies into a heptameric, wheel-like structure known as the apoptosome (Acehan et al., 2002; Kim et al., 2008). Oligomerized Apaf-1 recruits caspase- 9 through an interaction involving the caspase recruitment domain (CARD) on both proteins. Caspase- 9 is subsequently activated by oligomerization (Boatright et al., 2003). 


\section{PHYSIOLOGIC AND PATHOLOGICAL REGULATION OF CYTOCHROME C-MEDIATED CASPASE-9 ACTIVATION}

Regulation of cytochrome c-mediated caspase-9 activation occurs both before and after cytochrome $c$ release. Prior to cytochrome $c$ release, $\mathrm{Bcl}-2$ family proteins are major regulators of the apoptotic switch. These proteins, which contain up to four Bcl-2 homolog (BH1 to $\mathrm{BH} 4)$ domains, are divided into an anti-apoptotic subfamily (e.g., Bcl-2, Bcl-XL, and $\mathrm{Mcl}-1$ ) and two pro-apoptotic subfamilies: multiple $\mathrm{BH}$ domain-containing proteins (e.g., Bax and Bak) and BH3-only proteins (e.g., Bid) (Adams and Cory, 1998). BH3-only proteins act furthest upstream and are activated by various mechanisms including increased transcription, phosphorylation, and proteolytic processing (Huang and Strasser, 2000). While the precise mechanisms are unclear, BH3-only proteins promote cytochrome $c$ release either directly by stimulating multiple $\mathrm{BH}$ domain-containing proteins to form pores on the mitochondrial outer membrane or indirectly by counteracting anti-apoptotic $\mathrm{Bcl}-2$ proteins (Chipuk and Green, 2008).

A range of cellular factors regulates apoptosome formation after cytochrome $c$ release. The physiologic concentrations of potassium and calcium suppress apoptosome formation, the latter by preventing nucleotide exchange by Apaf-1 (Cain et al., 2001; Bao et al., 2007). HSP70 and HSP90 have been demonstrated to interact with Apaf-1 and inhibit interaction between Apaf-1 and caspase-9 (Beere et al., 2000; Bruey et al., 2000; Pandey et al., 2000; Saleh et al., 2000), while HSP27 binds to cytochrome $c$ and blocks its interaction with Apaf-1 (Bruey et al., 2000). The oncoprotein prothymosin- $\alpha$ (Pro-T) also impairs apoptosome assembly although the mechanism is undefined (Jiang et al., 2003). Furthermore, although low levels of dATP or ATP promote apoptosome formation, high levels of these nucleotides inhibit apoptosome by directly binding to cytochrome $c$ (Chandra et al., 2006).

Post-translational modification of cytochrome $c$ is critical for apoptosome formation. Newly synthesized cytochrome $c$ is unable to activate Apaf-1 until it undergoes heme attachment concurrent with its import into mitochondria (Martin and Fearnhead, 2002). Additionally, oxidized but not reduced cytochrome $c$ has been found to activate caspases (Saleh et al., 2000). In the physiological setting, intracellular glutathione (GSH), generated as a result of glucose metabolism by the pentose phosphate pathway, is necessary for maintaining cytochrome $c$ in a reduced form and abrogating its pro-apoptotic function (Vaughn and Deshmukh, 2008).

Cellular life and death decisions can be changed even after apoptosome formation. Cytosolic cytochrome c-mediated caspase activation is directly inhibited by the inhibitor of apoptosis (IAP) proteins. IAPs were originally identified in baculoviruses, and homologs are present throughout the tree of life. Each IAP protein contains at least one but often two to three characteristic zinc binding BIR sequences (baculovirus IAP repeats) (Salvesen and Duckett, 2002). In mammalian cells, X-linked IAP (XIAP) is a potent caspase inhibitor. XIAP binds to partially processed caspase- 9 and blocks its activity and further activation. XIAP also inhibits caspase-3 and caspase-7 (Salvesen and Duckett, 2002).

The intrinsic apoptotic pathway is frequently inactivated in tumor cells by a variety of mechanisms. Several of the more prominent examples are described here. The tumor suppressor p53 is probably the most commonly mutated gene in human cancer. Wild type p53 activates transcription of Bax and $\mathrm{BH}-3-$ only proteins (e.g., Puma, Noxa, and Bid), while also acting as a $\mathrm{BH} 3$-only protein to induce cytochrome $c$ release independently of transcription. Both functions are disabled by most, if not all, tumor-associated p53 mutations (Vousden and Lane, 2007). The Bcl-2 family is also widely dysregulated in tumors. The founding member of this group, $\mathrm{Bcl}-2$, was identified as a highly expressed oncogene in human follicular B cell lymphomas due to a chromosomal translocation that places $\mathrm{Bcl}-2$ adjacent to the $\lg \mathrm{G}$ heavy chain locus (Adams and Cory, 1998). Mcl-1 is another wellknown anti-apoptotic $\mathrm{Bcl}-2$ protein. This protein is rapidly degraded in normal cells, but its stability is increased in some tumor cells (Schwickart et al., 2010). Apaf-1 expression is decreased in some melanoma cells by hyper-methylation of the Apaf-1 promoter (Soengas et al., 2001). Another common mutation in tumor cells is the highly active PI-3K/Akt pathway. One target for this pathway is the BH3-only protein Bad, which upon phosphorylation forms a complex with 14-3-3 and loses its pro-apoptotic activity (Datta et al., 1997).

tRNA, and ribonucleic acid in general, is a recently identified and unique player in apoptotic regulation (Mei et al., 2010). Changes in the levels of tRNA and other RNAs are ubiquitous in oncogenic settings (Ruggero and Pandolfi, 2003; White, 2005). This raises the attractive possibility that RNAs play a pivotal role in the regulation of apoptosis.

\section{CANONICAL tRNA FUNCTION: THE ADAPTOR FOR PROTEIN SYNTHESIS}

tRNAs arose early in evolution as the adaptors in the translation of genetic information into protein sequences. All tRNA molecules fold into a conserved cloverleaf secondary structure through regions of internal self-complementarity. A set of conserved nucleotides then facilitates the formation of a compact L-shaped tertiary structure (Fig. 1). The folding of the $L$ shape involves coaxial stacking of the acceptor stem with the T stem-loop to form the top arm of the $L$, and coaxial stacking of the dihydrouridine stem (D-stem) with the anticodon stem-loop to form the vertical arm of the L. tRNAs are differentiated from each other according to the attached amino acid at the 3 ' end, which matches the anticodon triplet on the vertical arm. This match of an amino acid with a trinucleotide sequence in tRNA is the underlying basis of the 
genetic code. It is through this match that aminoacyl-tRNAs deliver amino acids to specific codon positions on the ribosome through base pairing interactions with tRNA anticodons, thus enabling decoding of mRNA sequences into amino acid sequences. The degeneracy of the genetic code, whereby more than one triplet codons correspond to a given amino acid, is achieved by having multiple tRNAs (known as isoacceptors) with the same amino acid specificity but with distinct anticodons.

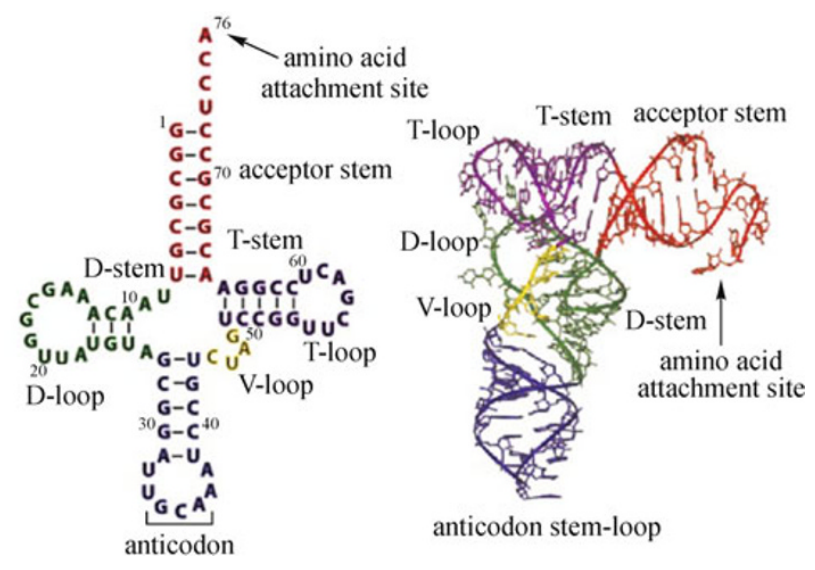

Figure 1. Sequence and structure of $E$. coli tRNA ${ }^{\text {Cys }}$ : (left) the cloverleaf secondary structure and (right) the Lshaped tertiary structure.

Many enzymes required for the tRNA adaptor function are both highly conserved and essential for growth. For example, all mature tRNAs possess the CCA sequence at the $3^{\prime}$ end as the obligate site for aminoacylation and for stable interaction with the ribosome (Fig. 1). This sequence is not encoded in human genomic DNA and must be added post-transcriptionally by the CCA-adding enzyme, which exists in all living organisms. The human CCA enzyme shares considerable sequence and structural homology with its bacterial counterparts (Yue et al., 1996). Aminoacylation of tRNA is catalyzed by aminoacyl-tRNA synthetases (aaRSs); there are 20 such enzymes in mammalian cells, one for each canonical amino acid, and these enzymes are highly conserved throughout their respective amino acid families.

The human mitochondrial genome encodes a separate set of tRNAs specifically for protein synthesis in the organelle. Although mitochondrial tRNAs generally have lower GC content and shorter stem-loop regions compared to their cytoplasmic counterparts, they still appear to fold into the same conserved L-shaped tertiary structure (de Bruijn and Klug, 1983; Watanabe et al., 1994). Importantly, protein enzymes that operate on mitochondrial tRNAs for the adaptor functions in the organelle, such as the mitochondrial CCA enzyme, and the 20 mitochondrial aminoacyl-tRNA synthetases are encoded by the nuclear genome. These nuclearencoded mitochondrial enzymes nonetheless share high sequence homology with their cytoplasmic counterparts. The strong conservation of protein components that enable and promote the tRNA adaptor function emphasizes the importance of this role in biology.

\section{NON-CANONICAL FUNCTIONS OF tRNA}

Several recent examples suggest that tRNAs have important extra-translational functions. First, replication of the RNA genome of human immunodeficiency virus 1 (HIV-1) requires the CCA sequence of the human host $\operatorname{tRNA}_{3}^{\text {Lys }}$ as the primer for initiation of the replication cycle (reviewed in Kleiman et al., 2010). The specific $\operatorname{tRNA}_{3}^{\text {Lys }}$, together with the other two tRNA ${ }^{\text {Lys }}$ isoacceptors and the aminoacylation enzyme lysyltRNA synthetase (LysRS), are also required for packaging of the virus. The molecular interactions that direct the assembly

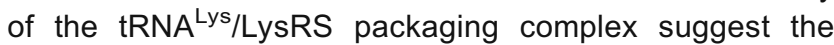
potential for developing new anti-viral agents. Second, while the translation initiator $\operatorname{tRNA}_{i}^{\text {Met }}$ plays a crucial role in the initiation of protein synthesis, it also has the ability to act as a pre-mRNA splicing regulator in a manner independent of its role in protein synthesis. Specifically, alternative splicing events resulting from AUG codon mutations are suppressible by initiator $t R N A_{i}^{M e t}$ variants harboring anticodon mutations that match the AUG mutations (Kamhi et al., 2010). This mechanism of regulation of splicing appears to play a role in quality control of splicing in the cell nucleus, preventing the generation of premature termination codons. Third, tRNA is a sensor of stress and nutrient deprivation that responds to these and other adverse situations by translocating in a retrograde fashion from the cytosol to the nucleus (Shaheen et al., 2007). This retrograde movement reduces tRNA availability in the cytosol, perhaps minimizing energy expenditure from protein synthesis. Uncharged tRNAs also accumulate in nutrient deprivation conditions and are efficient activators of the GCN2 kinase pathway (Hinnebusch, 2005). Activated GCN2 kinase phosphorylates the initiation factor elF2a, causing inactivation of the initiation factor elF2B, and repression of GCN4, an activator of general protein synthesis. This reduces the overall rate of protein synthesis, limiting amino acid consumption while allowing the cell to translate appropriate amino acid biosynthesis and stress-response genes.

Stress conditions also result in cleavage of tRNAs near anticodon sequences, resulting in tRNA half molecules (Thompson et al., 2008; Thompson and Parker, 2009a, b). These tRNA fragments have been identified in a wide variety of stress conditions, particularly during amino acid starvation or oxidative stress. Stress-induced tRNA cleavage may be catalyzed by a Dicer-dependent complex (Cole et al., 2009), or by the enzyme angiogenin (Yamasaki et al., 2009), a member of the RNase A family. Angionenin is typically sequestered in the nuclear compartment but is released into the cytosol during stress (Yamasaki et al., 2009). Production 
of tRNA fragments does not significantly deplete total tRNA levels, suggesting fragment generation has a function beyond depletion of cellular tRNA pools. One hypothesis is that tRNA fragments may inhibit global translation by forming repression complexes to block the initiation or elongation steps of protein synthesis (Yamasaki et al., 2009), or to affect the degradation or repression of specific mRNAs by recruiting tRNA processing enzymes to mRNAs (Elbarbary et al., 2009a, b). One tRNA fragment in particular has been implicated in proliferation of a broad range of cancer cells (Lee et al., 2009), indicating a role in tumor progression.

\section{THE CYTOCHROME C AND tRNA CONNECTION}

We began examining possible roles for RNA in regulating apoptosis many years ago. A puzzling observation was that up to $1 \mathrm{mM}$ concentration of dATP is needed to induce caspase- 9 activation in cell lysates while the intracellular concentration of dATP is only in $10 \mu \mathrm{M}$ range (Liu et al., 1996; Mesner et al., 1999). One possible explanation for this was that an inhibitor was present in the cell lysates that decreased the effectiveness of dATP. It seemed possible to us that RNA, essentially a polymer of nucleoside monophosphates, might have an inhibitory effect. This was supported by the observations that RNase treatment of S100 HeLa and Jurkat cell lysates strongly increased caspase- 9 activation, and that addition of total cellular RNA to either S100 extracts or a reconstituted caspase- 9 activation system potently blocked caspase- 9 activation. These results implicate an inhibitory role of RNA in the activation of caspase-9 (Mei et al., 2010).

Systematic examination of the steps leading to caspase- 9 cleavage identified cytochrome $c$ as the target of the RNA inhibitor. Analysis of cytochrome $c$-associated species found that it was tRNA that binds specifically to cytochrome $c$ both in vitro and in cells. The relevance of this finding in living cells was demonstrated by the ability of microinjected tRNA to inhibit cytochrome $c$-induced apoptosis, and by the ability of a tRNA-specific RNase to enhance both apoptosis and caspase-9 activation (Mei et al., 2010). Taken together, these results show that tRNA binds to cytochrome $c$ and inhibits the formation of the apoptosome (Fig. 2).

This finding raises intriguing questions regarding the generality of a role of tRNA in apoptotic and other protein functions. Below we discuss briefly the implications of this finding in the context of cell death, proliferation, and oncogenic transformation.

\section{ARE CYTOCHROME C AND tRNA LIVING AN AGE- OLD ROMANCE?}

Both cytochrome $c$ and tRNA are ancient molecules. tRNA is present in all known forms of life, and its secondary and tertiary structures are highly conserved across species and organelles (see above). Cytochrome $c$ is present in most eukaryotes that have a mitochondrial electron transport chain. Cytochrome $c$ from any species usually cross-reacts with cytochrome $c$-oxidase enzymes from another species, underscoring the conservation of cytochrome $c$ function throughout evolution. We speculate that the cytochrome $c$ :tRNA interaction is similarly evolutionarily ancient. Given the small size ( 100 aa) and highly positively charged nature of cytochrome $c$, its preferential binding to tRNA relative to other RNAs is striking. The structural nature of this association should be highly interesting.

Suppression of the cytochrome c's pro-apoptotic function by tRNA links protein synthesis to apoptotic susceptibility. Cells with high translation rates are often rapidly proliferating, and increased apoptotic resistance may prevent futile cycles of cellular proliferation and death. The cytochrome c:tRNA connection may also contribute to apoptotic resistance of tumor cells, which is supported by multiple lines of evidence. Human cytosolic tRNAs are transcribed by RNA polymerase III (pol III) (Lowe and Eddy, 1997; White, 2004). The critical tumor suppressors p53 and Rb directly bind and inhibit pol III, while c-Myc and Ras activate pol III transcription (Larminie et al., 1999; Crighton et al., 2003; Gomez-Roman et al., 2006). Increased biosynthesis of tRNA is required for proliferation and likely represents an obligatory step in tumorigenesis (White, 2005; Marshall et al., 2008). Indeed, tRNA is highly expressed in tumor cells (White, 2005).

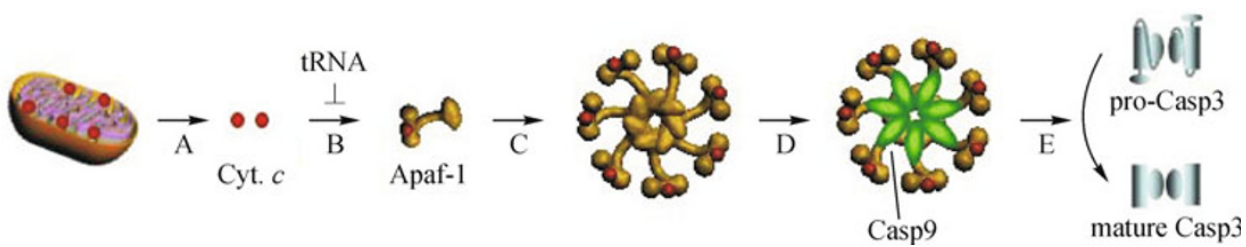

Figure 2. Cytochrome $c$-mediated caspase activation. Intracellular apoptotic stimuli provoke the release of cytochrome $c$ (cyt. c) from mitochondria to the cytosol (A), where it binds to Apaf-1 (B) promoting the assembly of Apaf-1 into the heptameric apoptosome (C). The apoptosome recruits and oligomerizes the precursors of caspase-9 (Casp9), leading to its auto-proteolytic processing (D). Mature caspase- 9 then activates procaspase-3 (pro-Casp3) through trans-cleavage (E). tRNA binds to cytochrome $c$ and prevents its interaction with Apaf-1. 
For example, breast cancer cells have 4-5 fold increased median nuclear tRNA expression and 5-20 fold increased median mitochondrial tRNA expression (Pavon-Eternod et al., 2009). Additionally, several RNase drugs have shown specific anti-tumor activity that is dependent on their catalytic ability (Costanzi et al., 2005). Onconase/Ranpirnase is the furthest developed and has reached phase III clinical trials for the lung tumor mesothelioma, and is in phase II trials for several other cancers (Costanzi et al., 2005). Onconase preferentially cleaves tRNA in a manner that correlates with apoptotic sensitivity (lordanov et al., 2000; Saxena et al., 2002; Suhasini and Sirdeshmukh, 2006). As a potential chemotherapeutic agent, Onconase has attractive attributes, including low systemic toxicity and p53independent killing. We believe that a better understanding of the role of tRNA in apoptotic resistance should provide a rational basis for the use and improvement of tRNA-based tumor therapy.

Relative to its role in electron transport, the ability of cytochrome $c$ to initiate apoptosis is a relatively recent evolutionary phenomenon. For example, cytochrome $c$ from C. elegans does not induce apoptosome formation or activate caspases (Riedl and Salvesen, 2007). Thus, the role of cytochrome $c$ in the electron transport chain is even more basic than its role in apoptosis. Cytochrome $c$ carries electrons from the mitochondrial inner membrane protein complex III to complex IV and is essential for the generation of the mitochondrial membrane potential $(\Delta \psi)$ that drives ATP formation. Interaction between cytochrome $c$ and both mitochondrial and cytosolic tRNA has been detected in healthy cells. It is thus possible that tRNA may regulate the electron transport chain and oxidative phosphorylation. It remains to be seen whether the cytochrome $c$ :tRNA interaction provides a way to coordinate protein translation both inside and outside mitochondria with energy production. An additional point of interest is that mutations in mitochondrial tRNA are widely associated with human diseases (Wallace, 2005). It is generally assumed that these mutations affect mitochondrial protein synthesis, although protein synthesis defects have not always been detected in such cases. It would be interesting to test whether these tRNA mutations might affect cytochrome $c$ action.

Cytochrome $c$ and tRNA are both ancient and extensively studied molecules. The surprising interaction between them underscores how much we have yet to learn about other wellstudied biologic systems. This interaction is a striking example of direct regulation of protein function by RNA that is very different from well-established models in which RNA acts principally at the level of gene expression. The protein and RNA worlds may be further intertwined than previously imagined.

\section{ACKNOWLEDGEMENTS}

We thank Lili Guo for designing Fig. 2. The work is supported by $\mathrm{NIH}$ grants GM60911 and CA088868 to X.Y. and GM68561 and GM81601 to Y.-M. H.

\section{ABBREVIATIONS}

aaRS, aminoacyl-tRNA synthetase; Apaf-1, the apoptotic protease activating factor-1; CARD, caspase recruitment domain; CAS, cellular apoptosis susceptibility protein; DISC, death inducing signaling complex; D-stem, dihydrouridine stem; GSH, glutathione; IAP, inhibitor of apoptosis; LysRS, lysyl-tRNA synthetase; MOMP, mitochondrial outer membrane permeabilization; PHAPI, putative HLA-DR-associated protein 1; pol III, RNA polymerase III; Pro-T, prothymosin- $\alpha$; XIAP, X-linked IAP

\section{REFERENCES}

Acehan, D., Jiang, X., Morgan, D.G., Heuser, J.E., Wang, X., and Akey, C.W. (2002). Three-dimensional structure of the apoptosome: implications for assembly, procaspase- 9 binding, and activation. Mol Cell 9, 423-432.

Adams, J.M., and Cory, S. (1998). The Bcl-2 protein family: arbiters of cell survival. Science 281, 1322-1326.

Ashkenazi, A., and Dixit, V.M. (1998). Death receptors: signaling and modulation. Science 281, 1305-1308.

Bao, Q., Lu, W., Rabinowitz, J.D., and Shi, Y. (2007). Calcium blocks formation of apoptosome by preventing nucleotide exchange in Apaf-1. Mol Cell25, 181-192.

Beere, H.M., Wolf, B.B., Cain, K., Mosser, D.D., Mahboubi, A., Kuwana, T., Tailor, P., Morimoto, R.I., Cohen, G.M., and Green, D. R. (2000). Heat-shock protein 70 inhibits apoptosis by preventing recruitment of procaspase- 9 to the Apaf-1 apoptosome. Nat Cell Biol 2, 469-475.

Boatright, K.M., Renatus, M., Scott, F.L., Sperandio, S., Shin, H., Pedersen, I.M., Ricci, J.E., Edris, W.A., Sutherlin, D.P., Green, D. R., et al. (2003). A unified model for apical caspase activation. Mol Cell 11, 529-541.

Bruey, J.M., Ducasse, C., Bonniaud, P., Ravagnan, L., Susin, S.A., Diaz-Latoud, C., Gurbuxani, S., Arrigo, A.P., Kroemer, G., Solary, E., et al. (2000). Hsp27 negatively regulates cell death by interacting with cytochrome c. Nat Cell Biol 2, 645-652.

Cain, K., Langlais, C., Sun, X.M., Brown, D.G., and Cohen, G.M. (2001). Physiological concentrations of $\mathrm{K}+$ inhibit cytochrome cdependent formation of the apoptosome. J Biol Chem 276, 41985-41990.

Chandra, D., Bratton, S.B., Person, M.D., Tian, Y., Martin, A.G., Ayres, M., Fearnhead, H.O., Gandhi, V., and Tang, D.G. (2006). Intracellular nucleotides act as critical prosurvival factors by binding to cytochrome $\mathrm{C}$ and inhibiting apoptosome. Cell 125, 1333-1346.

Chang, D.W., Xing, Z., Capacio, V.L., Peter, M.E., and Yang, X. (2003). Interdimer processing mechanism of procaspase-8 activation. EMBO J 22, 4132-4142.

Chang, H.Y., and Yang, X. (2000). Proteases for cell suicide: functions and regulation of caspases. Microbiol Mol Biol Rev 64, $821-846$.

Chipuk, J.E., and Green, D.R. (2008). How do BCL-2 proteins induce mitochondrial outer membrane permeabilization? Trends Cell Biol 18, 157-164.

Cole, C., Sobala, A., Lu, C., Thatcher, S.R., Bowman, A., Brown, J.W., 
Green, P.J., Barton, G.J., and Hutvagner, G. (2009). Filtering of deep sequencing data reveals the existence of abundant Dicerdependent small RNAs derived from tRNAs. RNA 15, 2147-2160.

Costanzi, J., Sidransky, D., Navon, A., and Goldsweig, H. (2005). Ribonucleases as a novel pro-apoptotic anticancer strategy: review of the preclinical and clinical data for ranpirnase. Cancer Invest 23, 643-650.

Crighton, D., Woiwode, A., Zhang, C., Mandavia, N., Morton, J.P., Warnock, L.J., Milner, J., White, R.J., and Johnson, D.L. (2003). p53 represses RNA polymerase III transcription by targeting TBP and inhibiting promoter occupancy by TFIIIB. EMBO J 22, 2810-2820.

Datta, S.R., Dudek, H., Tao, X., Masters, S., Fu, H., Gotoh, Y., and Greenberg, M.E. (1997). Akt phosphorylation of BAD couples survival signals to the cell-intrinsic death machinery. Cell 91, 231-241.

de Bruijn, M.H., and Klug, A. (1983). A model for the tertiary structure of mammalian mitochondrial transfer RNAs lacking the entire 'dihydrouridine' loop and stem. EMBO J 2, 1309-1321.

Elbarbary, R.A., Takaku, H., Uchiumi, N., Tamiya, H., Abe, M., Nishida, H., and Nashimoto, M. (2009a). Human cytosolic tRNase ZL can downregulate gene expression through miRNA. FEBS Lett 583, 3241-3246.

Elbarbary, R.A., Takaku, H., Uchiumi, N., Tamiya, H., Abe, M., Takahashi, M., Nishida, H., Nashimoto, M., and Randau, L. (2009b). Modulation of gene expression by human cytosolic tRNase Z(L) through 5'-half-tRNA. PLoS ONE 4, e5908.

Gomez-Roman, N., Felton-Edkins, Z.A., Kenneth, N.S., Goodfellow, S.J., Athineos, D., Zhang, J., Ramsbottom, B.A., Innes, F., Kantidakis, T., Kerr, E.R., et al. (2006). Activation by c-Myc of transcription by RNA polymerases I, II and III. Biochem Soc Symp, 141-154.

Hinnebusch, A.G. (2005). Translational regulation of GCN4 and the general amino acid control of yeast. Annu Rev Microbiol 59, 407-450.

Huang, D.C., and Strasser, A. (2000). BH3-Only proteins-essential initiators of apoptotic cell death. [In Process Citation] Cell 103, 839-842.

lordanov, M.S., Ryabinina, O.P., Wong, J., Dinh, T.H., Newton, D.L., Rybak, S.M., and Magun, B.E. (2000). Molecular determinants of apoptosis induced by the cytotoxic ribonuclease onconase: evidence for cytotoxic mechanisms different from inhibition of protein synthesis. Cancer Res 60, 1983-1994.

Jiang, X., Kim, H.E., Shu, H., Zhao, Y., Zhang, H., Kofron, J., Donnelly, J., Burns, D., Ng, S.C., Rosenberg, S., et al. (2003). Distinctive roles of PHAP proteins and prothymosin-alpha in a death regulatory pathway. Science 299, 223-226.

Kamhi, E., Raitskin, O., Sperling, R., and Sperling, J. (2010). A potential role for initiator-tRNA in pre-mRNA splicing regulation. Proc Natl Acad Sci U S A 107, 11319-11324.

Kim, H.E., Du, F., Fang, M., and Wang, X. (2005). Formation of apoptosome is initiated by cytochrome c-induced dATP hydrolysis and subsequent nucleotide exchange on Apaf-1. Proc Natl Acad Sci U S A 102, 17545-17550.

Kim, H.E., Jiang, X., Du, F., and Wang, X. (2008). PHAPI, CAS, and Hsp70 promote apoptosome formation by preventing Apaf-1 aggregation and enhancing nucleotide exchange on Apaf-1. Mol Cell 30, 239-247.
Kleiman, L., Jones, C.P., and Musier-Forsyth, K. (2010). Formation of the tRNALys packaging complex in HIV-1. FEBS Lett 584, 359-365.

Larminie, C.G., Sutcliffe, J.E., Tosh, K., Winter, A.G., Felton-Edkins, Z.A., and White, R.J. (1999). Activation of RNA polymerase III transcription in cells transformed by simian virus 40 . Mol Cell Biol 19, 4927-4934.

Lee, Y.S., Shibata, Y., Malhotra, A., and Dutta, A. (2009). A novel class of small RNAs: tRNA-derived RNA fragments (tRFs). Genes Dev 23, 2639-2649.

$\mathrm{Li}$, J., and Yuan, J. (2008). Caspases in apoptosis and beyond. Oncogene 27, 6194-6206.

Liu, X., Kim, C.N., Yang, J., Jemmerson, R., and Wang, X. (1996). Induction of apoptotic program in cell-free extracts: requirement for dATP and cytochrome c. Cell 86, 147-157.

Lowe, T.M., and Eddy, S.R. (1997). tRNAscan-SE: a program for improved detection of transfer RNA genes in genomic sequence. Nucleic Acids Res 25, 955-964.

Marshall, L., Kenneth, N.S., and White, R.J. (2008). Elevated tRNA (iMet) synthesis can drive cell proliferation and oncogenic transformation. Cell 133, 78-89.

Martin, A.G., and Fearnhead, H.O. (2002). Apocytochrome c blocks caspase- 9 activation and Bax-induced apoptosis. J Biol Chem 277, 50834-50841.

Mei, Y., Yong, J., Liu, H., Shi, Y., Meinkoth, J., Dreyfuss, G., and Yang, X. (2010). tRNA binds to cytochrome $c$ and inhibits caspase activation. Mol Cell 37, 668-678.

Mesner, P.W. Jr, Bible, K.C., Martins, L.M., Kottke, T.J., Srinivasula, S. M., Svingen, P.A., Chilcote, T.J., Basi, G.S., Tung, J.S., Krajewski, S., et al. (1999). Characterization of caspase processing and activation in HL-60 cell cytosol under cell-free conditions. Nucleotide requirement and inhibitor profile. J Biol Chem 274, 22635-22645.

Pandey, P., Saleh, A., Nakazawa, A., Kumar, S., Srinivasula, S.M., Kumar, V., Weichselbaum, R., Nalin, C., Alnemri, E.S., Kufe, D., et al. (2000). Negative regulation of cytochrome c-mediated oligomerization of Apaf-1 and activation of procaspase- 9 by heat shock protein 90. EMBO J 19, 4934-4322.

Pavon-Eternod, M., Gomes, S., Geslain, R., Dai, Q., Rosner, M.R., and Pan, T. (2009). tRNA over-expression in breast cancer and functional consequences. Nucleic Acids Res 37, 7268-7280.

Riedl, S.J., and Salvesen, G.S. (2007). The apoptosome: signalling platform of cell death. Nat Rev Mol Cell Biol 8, 405-413.

Ruggero, D., and Pandolfi, P.P. (2003). Does the ribosome translate cancer? Nat Rev Cancer 3, 179-192.

Saleh, A., Srinivasula, S.M., Balkir, L., Robbins, P.D., and Alnemri, E. S. (2000). Negative regulation of the Apaf-1 apoptosome by Hsp70. Nat Cell Biol 2, 476-483.

Salvesen, G.S., and Duckett, C.S. (2002). IAP proteins: blocking the road to death's door. Nat Rev Mol Cell Biol 3, 401-410.

Saxena, S.K., Sirdeshmukh, R., Ardelt, W., Mikulski, S.M., Shogen, K., and Youle, R.J. (2002). Entry into cells and selective degradation of tRNAs by a cytotoxic member of the RNase A family. J Biol Chem 277, 15142-15146.

Schwickart, M., Huang, X., Lill, J.R., Liu, J., Ferrando, R., French, D. M., Maecker, H., O'Rourke, K., Bazan, F., Eastham-Anderson, J., et al. (2010). Deubiquitinase USP9X stabilizes MCL1 and promotes tumour cell survival. Nature 463, 103-107. 
Shaheen, H.H., Horetsky, R.L., Kimball, S.R., Murthi, A., Jefferson, L. S., and Hopper, A.K. (2007). Retrograde nuclear accumulation of cytoplasmic tRNA in rat hepatoma cells in response to amino acid deprivation. Proc Natl Acad Sci U S A 104, 8845-8850.

Soengas, M.S., Capodieci, P., Polsky, D., Mora, J., Esteller, M., OpitzAraya, X., McCombie, R., Herman, J.G., Gerald, W.L., Lazebnik, Y. A., et al. (2001). Inactivation of the apoptosis effector Apaf-1 in malignant melanoma. Nature 409, 207-211.

Suhasini, A.N., and Sirdeshmukh, R. (2006). Transfer RNA cleavages by onconase reveal unusual cleavage sites. J Biol Chem 281, 12201-12209.

Thompson, C.B. (1995). Apoptosis in the pathogenesis and treatment of disease. Science 267, 1456-1462.

Thompson, D.M., Lu, C., Green, P.J., and Parker, R. (2008). tRNA cleavage is a conserved response to oxidative stress in eukaryotes. RNA 14, 2095-2103.

Thompson, D.M., and Parker, R. (2009a). The RNase Rny1p cleaves tRNAs and promotes cell death during oxidative stress in Saccharomyces cerevisiae. J Cell Biol 185, 43-50.

Thompson, D.M., and Parker, R. (2009b). Stressing out over tRNA cleavage. Cell 138, 215-219.

Vaughn, A.E., and Deshmukh, M. (2008). Glucose metabolism inhibits apoptosis in neurons and cancer cells by redox inactivation of cytochrome c. Nat Cell Biol 10, 1477-1483.

Vaux, D.L., and Korsmeyer, S.J. (1999). Cell death in development. Cell 96, 245-254.

Vousden, K.H., and Lane, D.P. (2007). p53 in health and disease. Nat Rev Mol Cell Biol 8, 275-283.
Wallace, D.C. (2005). A mitochondrial paradigm of metabolic and degenerative diseases, aging, and cancer: a dawn for evolutionary medicine. Annu Rev Genet 39, 359- 407.

Wang, X. (2001). The expanding role of mitochondria in apoptosis. Genes Dev 15, 2922-2933.

Watanabe, Y., Kawai, G., Yokogawa, T., Hayashi, N., Kumazawa, Y., Ueda, T., Nishikawa, K., Hirao, I., Miura, K., and Watanabe, K. (1994). Higher-order structure of bovine mitochondrial tRNA (SerUGA): chemical modification and computer modeling. Nucleic Acids Res 22, 5378-5384.

White, R.J. (2004). RNA polymerase III transcription and cancer. Oncogene 23, 3208-3216.

White, R.J. (2005). RNA polymerases I and III, growth control and cancer. Nat Rev Mol Cell Biol 6, 69-78.

Yamasaki, S., Ivanov, P., Hu, G.F., and Anderson, P. (2009). Angiogenin cleaves tRNA and promotes stress-induced translational repression. J Cell Biol 185, 35-42.

Yang, X., Chang, H.Y., and Baltimore, D. (1998). Autoproteolytic activation of pro-caspases by oligomerization. Mol Cell 1, 319-325.

Yue, D., Maizels, N., and Weiner, A.M. (1996). CCA-adding enzymes and poly $(A)$ polymerases are all members of the same nucleotidyltransferase superfamily: characterization of the CCA-adding enzyme from the archaeal hyperthermophile Sulfolobus shibatae. RNA 2, 895-908.

Zou, H., Henzel, W.J., Liu, X., Lutschg, A., and Wang, X. (1997). Apaf1 , a human protein homologous to $C$. elegans CED-4, participates in cytochrome c-dependent activation of caspase-3. Cell 90, 405- 413 . 